\title{
CAMLOG
}

\section{Markteinführung CAMLOG® Scankörper}

Für die präzise softwaregestützte Konstruktion von individuellen Abutments, Kronen- und Brückengerüsten auf der CAMLOG ${ }^{\oplus}$ Titanbasis CAD/CAM steht ab sofort der CAMLOG ${ }^{\circledR}$ Scankörper zur Verfügung.

Der Scankörper aus dem widerstandsfähigen Kunststoff PEEK besitzt einen Implantat-Anschluss mit der bewährt zuverlässigen Tube-in-Tube ${ }^{\text {TM }}$ Implantat/Abutment-Verbindung. Dadurch ist die exakte und schnelle Positionierung auf den Laborimplantaten selbst bei mehrmaliger Wiederverwendung gewährleistet. Die eindeutig erfassbare Geometrie (bisher getestet mit 3ShapeScannern; Kompatibilität mit weiteren Scan-/CAD-Systemen in Vorbereitung), sehr geringe Fertigungstoleranzen und die verschraubte Anwendung resultieren in der präzisen digitalen Erfassung der Implantatlage - inklusive der Achsneigung und der Ausrichtung der Nuten.

Der neue CAMLOG ${ }^{\circledR}$ Scankörper ist für alle CAMLOG Implantat-Durchmesser erhältlich, entsprechend beschriftet und wird unsteril mit einer Laborschraube geliefert. Bisher ist er mit den Laborscannern und "offenen" Installationen der CAD-Software AbutmentDesigner ${ }^{\mathrm{TM}}$ von 3Shape A/S (Kopenhagen, DK) verwendbar. CAMLOG arbeitet sukzessive an der Erweiterung der Kompatibilität mit weiteren Systemen und wird darüber auf www.camlog.de/titanbasen informieren.

$\boldsymbol{\lambda}$ Tel. 07044 - 9445-100

info.de@camlog.com

www.camlog.de

\section{WDL Kindermöbel}

\section{Eine kindgerechte Praxiseinrichtung nimmt Ängste}

Um Kindern die Wartezeit vor einer Behandlung angenehmer zu gestalten, hat der Kindermöbelspezialist WDL aus dem Nordschwarzwald mit Triolino eine zeitlos harmonische Kombi aus einer Bank, zwei Hockern und einem Tisch entworfen, bei der alles mit wenigen Handgriffen in der Höhe verstellt werden kann. Die rötliche Farbe des massiven Erlenholzes strahlt Wärme und Zuversicht aus, die Kanten sind kindergerecht und zur
Sicherheit leicht abgerundet. Durch das robuste Material kann Triolino auch in stark frequentierten Praxen viele Jahre seinen Dienst leisten. Ein wenig Schleifen und Nachölen reicht, wenn sie doch einmal verschrammt sind. Wichtig: Alle Kindermöbel von WDL sind selbstverständlich TÜV-geprüft und werden regelmäßig nach strengen Kriterien auf Schadstoffe getestet. Vorbildlich ist auch der ökologisch-soziale Hintergrund: WDL Kindermöbel

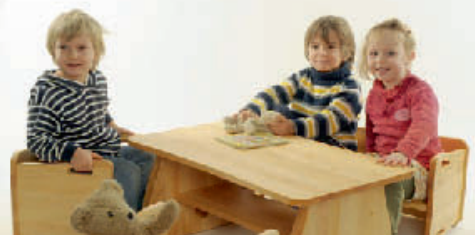

werden ausschließlich im Nordschwarzwald in einer Werkstatt für behinderte Menschen gefertigt.

入 Tel. 07221 - 989-111 a.bartele@wdl-ggmbh.de www.wdl-kinder-moebel.de

\section{CompuGroup Medical Dentalsysteme}

\section{Spracherkennung für Z1}

Mit der Entwicklung einer sprecherunabhängigen Spracherkennung unterstreicht die CompuGroup Medical Dentalsysteme $\mathrm{GmbH}$, Koblenz, einmal mehr ihre Kompetenz in Sachen innovativer Softwarelösungen.

Besonders hilfreich zeigt sich dieses Modul bei der Erfassung von PAR- oder 01-Befunden sowie bei assistenzloser IP oder PSI, denn die Hände bleiben stets frei für die Arbeit am Patienten. Unkom- pliziert und sicher werden Befundeingabe und -dokumentation über das Mikrofon eines Headsets gesteuert. Das neue Modul erkennt ohne aufwändiges Training alle gängigen Befund-Varianten und ignoriert dabei zuverlässig das "normale“ Patientengespräch.

Fazit: Z1-Anwender können nach ihren eigenen Angaben mit Hilfe der Spracherkennung unabhängiger und effektiver arbeiten. Einfacher geht es nicht!

入 Tel. 0261 - 8000-1900 info@cgm-dentalsysteme.de www.cgm-dentalsysteme.de 